\title{
Guaranteed Robust Nonlinear State Estimator with Application to Global Vehicle Tracking
}

\author{
M. Kieffer, E. Seignez, A. Lambert, E. Walter and T. Maurin
}

\begin{abstract}
This paper deals with guaranteed recursive state estimation in a bounded-error context with application to global dynamical vehicle tracking. As in Kalman or approximate Bayesian filtering, prediction and correction phases alternate. A distinctive feature of the method advocated here is that its results are guaranteed, in the sense that the statements made about the possible values of the state vector are mathematically proved, although all calculations are performed approximately on a computer. Sets will thus be provided that are guaranteed to contain all values of the state that are consistent with the information available and the bounds assumed on the state perturbations and measurement errors. Complexity issues are addressed and some tools are provided to facilitate real-time implementation. Results obtained with an actual vehicle are reported.
\end{abstract}

\section{INTRODUCTION}

Consider a system described by the discrete-time state equation

$$
\mathbf{x}_{k+1}=\mathbf{f}_{k}\left(\mathbf{x}_{k}, \mathbf{w}_{k}\right), \quad k=0,1, \ldots
$$

where $\mathbf{f}_{k}$ is a known function (possibly nonlinear and timevarying), $\mathbf{x}_{k}$ is the unknown state vector at time $k$ and $\mathbf{w}_{k}$ is some unknown state perturbation vector. Assume $\mathbf{x}_{k}$ is to be estimated based on past measurements satisfying

$$
\mathbf{y}_{\ell}=\mathbf{h}_{\ell}\left(\mathbf{x}_{\ell}, \mathbf{v}_{\ell}\right), \quad \ell=1, \ldots, k,
$$

where $\mathbf{h}_{\ell}$ is a known function (also possibly nonlinear and time-varying), $\mathbf{y}_{\ell}$ is the measurement vector at time $\ell$ and $\mathbf{v}_{\ell}$ is some unknown measurement noise vector. Depending on the nature of $\mathbf{f}_{k}$ and $\mathbf{h}_{\ell}$ and on the information assumed available about the state perturbation and measurement noise, various types of state estimators are available. If $\left\{\mathbf{w}_{k}, k \in \mathbb{N}\right\}$ and $\left\{\mathbf{v}_{k}, k \in \mathbb{N}\right\}$ are i.i.d. sequences with known probability density functions (pdf) and if the pdf of $\mathbf{x}_{0}$ based on no measurement is known, then it becomes possible, at least in principle, recursively to compute $p\left(\mathbf{x}_{k} \mid \mathbf{y}_{1: k}\right)$, the posterior pdf of $\mathbf{x}_{k}$ based on the first $k$ measurements, thus providing the optimal solution of the state estimation problem in a Bayesian sense. This is achieved by alternating predictions during which the prior pdf $p\left(\mathbf{x}_{k} \mid \mathbf{y}_{1: k-1}\right)$ is computed via the ChapmanKolgomorov equation and corrections during which the new

This work was partly supported by PPF VARVIC

M. Kieffer and E. Walter are with the Laboratoire des Signaux et Systèmes - CNRS - Supélec - Université Paris-Sud, Plateau de Moulon, F-91192 Gif-sur-Yvette, France name@lss.supelec.fr

E. Seignez, A. Lambert and T. Maurin are with the Institut d'Électronique Fondamentale - CNRS - Université Paris-Sud, F-91400 Orsay, France namedief.u-psud.fr measurement $\mathbf{y}_{k}$ is taken into account to update the prior pdf into the posterior pdf $p\left(\mathbf{x}_{k} \mid \mathbf{y}_{k}\right)$ via Bayes' rule.

If $\mathbf{f}_{k}$ and $\mathbf{h}_{\ell}$ are linear and the probability density functions of $\mathbf{x}_{0}$ and the state perturbation and measurement noise are Gaussian with known means and covariances, then this optimal solution is provided by the Kalman filter [20]. For the general nonlinear case, however, no such explicit solution is available, and one has to resort to approximations. A first such approximation is extended Kalman filtering [1], [7], based on a linearization of the model around its estimated trajectory in state space. The fact that this approach cannot accurately describe situations where the model is highly nonlinear and the pdf of the state is far from Gaussian has motivated the development of grid-based methods [4], [21], where the state space is partitioned a priori into cells and integrals are replaced by discrete approximations. This approach lacks flexibility, as it does not allow the partition to be adapted dynamically so as to get more resolution in regions with high probability. The very popular particle filtering methods [2], [8], [15], [18] do not have this limitation. They implement Bayesian filtering by Monte-Carlo simulation, representing pdf by sets of support points (particles) with associated weights (masses). Various heuristics need to be implemented to eliminate particles with masses that become neglectable while preserving the diversity of the population, and no guarantee can be provided as to the quality of the results to be obtained.

An attractive alternative to these approaches is boundederror state estimation, also known as guaranteed state estimation. Guaranteed means here that, under some explicitly stated hypotheses, the statements to be made about the possible values of the state vector will be mathematically proved, although all calculations are performed with finite accuracy on a computer.

After recalling briefly a methodology based on interval analysis for bounded-error nonlinear state estimation [12], we shall apply it to an actual problem of vehicle tracking.

The specific application considered is the estimation of the configuration (position and orientation) of the vehicle. The information available is a map of the environment and distances to obstacles which are periodically measured by onboard sonars. This problem is challenging, because

- the initial configuration of the vehicle is not assumed to be known,

- the state vector may not be observable,

- the state equation of the vehicle is nonlinear and uncertain, 
- the measurement equation is strongly nonlinear,

- the precision of the sensors is low,

- a large proportion of the data points turn out to be outliers.

An additional advantage of this problem is that it has been used as a test-case for most of the methods available for state estimation. (Extended) Kalman filtering has been employed [3], [5], but requires a fairly accurate knowledge of the initial configuration of the vehicle. Moreover, due to linearizations of the state and observation equations, the actual configuration may get lost during recursions. To achieve an initial localization or to track several possible configurations in ambiguous situations, probability grids have been used in [4], [19]. Particle filtering has been applied to global localization, e.g., in [6], [22].

In all of these approaches, the state perturbations and measurement noise are either assumed Gaussian or approximately characterized by their first two moments. We shall assume instead that they are bounded with known bounds (while allowing the presence of outliers, i.e., data points for which the measurement error is much larger than allowed by these bounds). Our aim is then the guaranteed characterization of the set of all the state vectors that are consistent with the measurements and this hypothesis. So far, this was mostly achieved in simulations [10], with experimentation limited to initial localization [16].

Section II briefly recalls the principles of nonlinear discrete-time bounded-error state estimation based on interval analysis. Global indoor localization and tracking of a vehicle from ultrasonic range measurements is described in Section III. Section IV addresses the complexity of the proposed algorithms and provides some tools to facilitate their real-time implementation. Experimental results are reported in Section V.

\section{State Estimation USING Interval ANALYsis}

This section briefly recalls the principles of recursive discrete-time bounded-error state estimation using interval analysis. For more details, see [9], [12], [13].

\section{A. Introduction}

Consider a nonlinear and possibly time-varying system described by

$$
\left\{\begin{array}{l}
\mathbf{x}_{k+1}=\mathbf{f}_{k}\left(\mathbf{x}_{k}, \mathbf{w}_{k}\right), k=0,1, \ldots \\
\mathbf{y}_{k}=\mathbf{h}_{k}\left(\mathbf{x}_{k}\right)+\mathbf{v}_{k}, k=1,2, \ldots
\end{array}\right.
$$

where $\mathbf{x}_{k} \in \mathbb{R}^{n}$ and $\mathbf{y}_{k} \in \mathbb{R}^{p}$ are respectively the state and output vectors. The observation equation in (3) is a special case of (2), as the observation noise is assumed additive for the sake of simplicity. The initial state $\mathbf{x}_{0}$ is now assumed to belong to some prior compact set $\mathcal{X}_{0} \subset$ $\mathbb{R}^{n}$, and $\mathbf{w}_{k}$ and $\mathbf{v}_{k}$ are respectively assumed to belong to the known interval vectors (or boxes) $[\mathbf{w}]$ and $[\mathbf{v}]$. The problem to be considered is the recursive characterization of the smallest set $\mathcal{X}_{k}$ guaranteed to contain all values of $\mathbf{x}_{k}$ compatible with the information available just after the $k$-th measurement, i.e., with

$$
\mathcal{I}_{k}=\left\{\mathcal{X}_{0},[\mathbf{w}],[\mathbf{v}],\left\{\mathbf{y}_{\ell}\right\}_{\ell=1}^{k}\right\} .
$$

A two-step recursive algorithm similar to the Kalman filter can be built that computes outer approximations $\widehat{\mathcal{X}}_{k}$ of $\mathcal{X}_{k}$ at each $k$. This algorithm alternates prediction and correction and requires some techniques from interval analysis to be implemented.

With a notation similar to that usual in Kalman filtering, we shall denote the set of all possible values of $\mathbf{x}_{k}$ based on measurements up to time $k-1$ by $\mathcal{X}_{k \mid k-1}$. This set is updated into $\mathcal{X}_{k \mid k}$ when $\mathbf{y}_{k}$ is taken into account.

\section{B. Interval analysis}

Interval analysis provides a set of tools to compute with intervals $[x]=[\underline{x}, \bar{x}] \subset \mathbb{R}$ and vector of intervals (or boxes) $[\mathbf{x}]=[\underline{\mathbf{x}}, \overline{\mathbf{x}}] \subset \mathbb{R}^{n}$. For any function $f: \mathcal{D} \subset \mathbb{R} \longrightarrow$ $\mathbb{R}$ defined as combinations of arithmetical operators and elementary functions, interval analysis makes it possible to build inclusion functions $[f]$ satisfying

$$
\forall[x] \subset \mathcal{D}, f([x]) \subset[f]([x]),
$$

where $[f]([x])$ is an interval. For example, the natural inclusion function is obtained by replacing all occurrences of a real variable by its interval counterpart. It thus becomes possible to enclose the set of all values taken by a function over a given interval into a computable image interval. If the width of this image interval tends to zero when the width of the corresponding argument interval tends to zero, then the inclusion functions is convergent, a desirable property usually satisfied under mild continuity constraints. For more details, see [17].

Compact sets are easily described by unions of nonoverlapping boxes or subpavings. This concept will be particularly useful to represent the sets computed during the prediction and correction steps.

\section{Prediction step}

If $\widehat{\mathcal{X}}_{k-1 \mid k-1}$ satisfying $\mathcal{X}_{k-1 \mid k-1} \subset \widehat{\mathcal{X}}_{k-1 \mid k-1}$ is available at time $k-1$, an outer approximation $\overline{\mathcal{X}}_{k \mid k-1}$ of the set $\mathcal{X}_{k \mid k-1}$ of all values of $\mathbf{x}_{k}$ that are consistent with $\mathcal{I}_{k-1}$ may be evaluated at time $k$ as

$$
\begin{aligned}
\overline{\mathcal{X}}_{k \mid k-1} & =\left\{\mathbf{f}_{k}(\mathbf{x}, \mathbf{w}) \text { with } \mathbf{x} \in \widehat{\mathcal{X}}_{k-1 \mid k-1}, \mathbf{w} \in[\mathbf{w}]\right\} \\
& =\mathbf{f}_{k}\left(\widehat{\mathcal{X}}_{k-1 \mid k-1},[\mathbf{w}]\right) .
\end{aligned}
$$

When $\widehat{\mathcal{X}}_{k-1 \mid k-1}$ is described by a subpaving and provided that an inclusion function for $\mathbf{f}_{k}$ is available, an outerapproximation $\widehat{\mathcal{X}}_{k \mid k-1}$ for $\overline{\mathcal{X}}_{k \mid k-1}$ may be evaluated using the IMAGESP algorithm [9]. IMAGESP consists of three steps.

- First, the subpaving $\widehat{\mathcal{X}}_{k-1 \mid k-1}$ is minced, i.e., its boxes are bisected until their width is lower than some specified precision parameter $\varepsilon_{\mathrm{I}}$. 
- The images of the resulting boxes are then evaluated using the inclusion function for $\mathbf{f}_{k}$. According to (5), the union of these images is guaranteed to contain $\mathbf{f}_{k}\left(\widehat{\mathcal{X}}_{k-1 \mid k-1},[\mathbf{w}]\right)$.

- Finally, these images are merged to get a subpaving $\widehat{\mathcal{X}}_{k \mid k-1}$ guaranteed to contain $\overline{\mathcal{X}}_{k \mid k-1}$ and thus $\mathcal{X}_{k \mid k-1}$.

Mincing allows a better estimation of the image to be obtained. When $\varepsilon_{\mathrm{I}}$ decreases, the image subpaving $\widehat{\mathcal{X}}_{k \mid k-1}$ gets closer to $\mathcal{X}_{k \mid k-1}$ (in the sense of the Hausdorf distance). The price to be paid is an increased computing time.

The last step of IMAGESP could be dispensed with. It allows a subpaving to be built from a collection of possibly overlapping boxes. This step reduces the number of boxes that have to be maintained and gives them some structure, which facilitates further computation.

\section{Correction step}

Assume that $\widehat{\mathcal{X}}_{k \mid k-1}$ satisfying $\mathcal{X}_{k \mid k-1} \subset \widehat{\mathcal{X}}_{k \mid k-1}$ has been obtained during the prediction step. The new output $\mathbf{y}_{k}$, made available at time $k$, can now be taken into account by updating $\widehat{\mathcal{X}}_{k \mid k-1}$ into an outer approximation $\widehat{\mathcal{X}}_{k \mid k}$ of the set

$$
\begin{aligned}
\overline{\mathcal{X}}_{k \mid k}= & \left\{\mathbf{x} \in \widehat{\mathcal{X}}_{k \mid k-1} \mid \mathbf{y}_{k}=\mathbf{h}_{k}(\mathbf{x})+\mathbf{v}, \mathbf{v} \in[\mathbf{v}]\right\} \\
= & \left\{\mathbf{x} \in \widehat{\mathcal{X}}_{k \mid k-1} \mid \mathbf{h}_{k}(\mathbf{x}) \in\left[\mathbf{y}_{k}\right]\right\} \\
& \text { with }\left[\mathbf{y}_{k}\right]=\left[\mathbf{y}_{k}-\overline{\mathbf{v}}, \mathbf{y}_{k}-\mathbf{v}\right] \\
= & \mathbf{h}_{k}^{-1}\left(\left[\mathbf{y}_{k}\right]\right) \cap \widehat{\mathcal{X}}_{k \mid k-1} .
\end{aligned}
$$

As $\mathcal{X}_{k \mid k-1} \subset \widehat{\mathcal{X}}_{k \mid k-1}, \mathcal{X}_{k \mid k} \subset \overline{\mathcal{X}}_{k \mid k}$. Since $\widehat{\mathcal{X}}_{k \mid k-1}$ is described by a union of boxes, $\widehat{\mathcal{X}}_{k \mid k}$ may be obtained as a union of boxes using the SIVIA algorithm [9]. SIVIA applies recursively the following tests to all boxes $[\mathbf{x}]$ of $\widehat{\mathcal{X}}_{k \mid k-1}$ :

- If $\left[\mathbf{h}_{k}\right]([\mathbf{x}]) \subset\left[\mathbf{y}_{k}\right]$, then according to (5), for all $\mathbf{x} \in[\mathbf{x}], \mathbf{h}_{k}(\mathbf{x}) \in\left[\mathbf{y}_{k}\right]$. Any $\mathbf{x} \in[\mathbf{x}]$ is thus consistent with the measurements and noise bounds and $[\mathbf{x}]$ is proved to be in $\widehat{\mathcal{X}}_{k \mid k}$.

- If $\left[\mathbf{h}_{k}\right]([\mathbf{x}]) \cap\left[\mathbf{y}_{k}\right]=\emptyset$, then, again according to (5), for all $\mathbf{x} \in[\mathbf{x}], \mathbf{h}_{k}(\mathbf{x}) \notin\left[\mathbf{y}_{k}\right]$. Therefore, there is no $\mathbf{x}$ in $[\mathbf{x}]$ that is consistent with the measurements and noise bounds and $[\mathbf{x}]$ does not belong to $\widehat{\mathcal{X}}_{k \mid k}$.

- In all other cases, if $w([\mathbf{x}])$ is larger than some specified precision parameter $\varepsilon_{\mathbf{S}}$, it is bisected across any of its dimension of larger width to get two subboxes on which the same tests are applied. If $w([\mathbf{x}])<\varepsilon_{\mathbf{S}}$, $[\mathbf{x}]$ is stored in $\widehat{\mathcal{X}}_{k \mid k}$.

\section{Application to Vehicle localization}

This section describes the experimental platform and the information available for global localization.

\section{A. Experimental platform}

Minitruck is represented on Figure 1. Built on a model of a truck at the $1 / 20$ scale, it is $25 \mathrm{~cm}$ large and $50 \mathrm{~cm}$ long. Low consumption electronics allows two to three hours of experimentation and the embedded computing power
(Xscale processor at $400 \mathrm{Mhz}$ ) is sufficient to implement onboard basic tasks like collision avoidance, Kalman filtering localisation, path tracking, automatic parking, etc. Tasks needing more computing power, like particle filtering

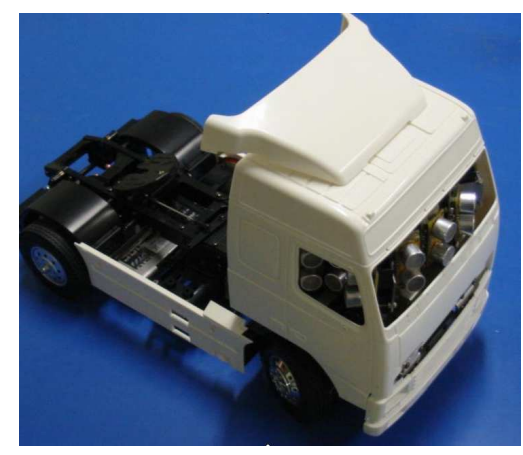

Fig. 1. Minitruck

and bounded-error localisation, path planning or mapping, can be deported on a distant computer using a client-server architecture. In this case, the exchanged information reduces to the sensor data from the experimental platform and the algorithmic results from the distant computer.

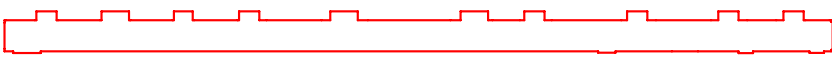

Fig. 2. Map of the environment of Minitruck

Minitruck is placed in a corridor represented on Figure 2. The map is defined by a collection of $n_{\mathrm{W}}$ oriented segments $\left[\mathbf{a}_{i}, \mathbf{b}_{i}\right]$ describing the environment.

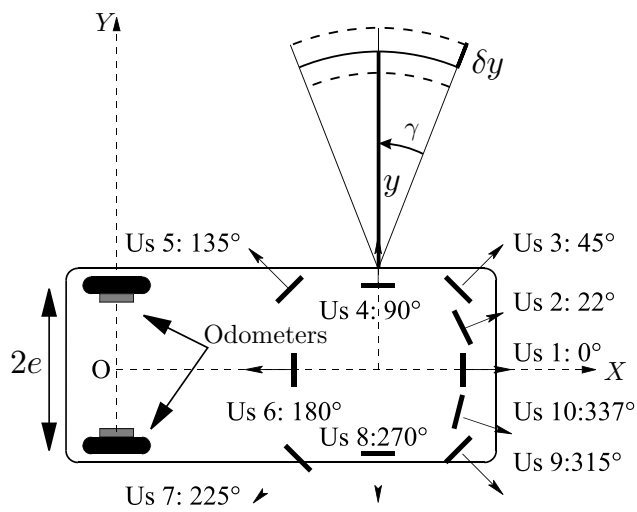

Fig. 3. Location of the sensors and uncertainty of the sonar measurements

Minitruck is equipped with two odometers (incremental coders) and $n_{\mathrm{S}}=10$ ultrasonic sensors. The odometers, which are mounted on each rear wheel, have an angular resolution of $0.18^{\circ}$ and achieve millimetric precision. Each of the ultrasonic sensors (Figure 3) measures twice per second the distance to the closest obstacle in its emission cone which has a half aperture $\gamma$ of $22^{\circ}$. Experimentation showed that these sonars are able to detect objects in a range 
between $3 \mathrm{~cm}$ and $6 \mathrm{~m}$. The additive uncertainty $\Delta y$ on a distance measurement $y$ is $\alpha=3 \%$ of the result.

\section{B. Models}

The configuration of Minitruck is given by $\mathbf{x}=$ $\left(x_{c}, y_{c}, \theta_{c}\right)^{\mathrm{T}}$, where $\left(x_{c}, y_{c}\right)$ is the position of the middle $\mathrm{O}$ of the segment joining the two rear wheels and $\theta_{c}$ gives the orientation of Minitruck in a global frame. The control inputs are the angle of the two front wheels and the mean speed of the rear wheels, or equivalently the vector $\mathbf{u}_{k}=\left(\delta \theta_{r}, \delta \theta_{l}\right)^{\mathrm{T}}$ representing the angular displacements of the right and left rear wheels during a time period $\delta t$. To take slipping into account, a bounded additive error $\Delta \mathbf{u}_{k}=\left(\Delta u_{k, l}, \Delta u_{k, r}\right)^{\mathrm{T}} \in\left[-\beta \mathbf{u}_{k}, \beta \mathbf{u}_{k}\right]$ on $\mathbf{u}_{k}$ is incorporated under the form of a state perturbation. From these displacements it is possible to deduce the incremental longitudinal motion $\delta s$ and rotational motion $\delta \theta$ of the vehicle

$$
\begin{aligned}
\delta s & =\frac{r}{2}\left(\delta \theta_{l}+\Delta u_{k, l}+\delta \theta_{r}+\Delta u_{k, r}\right) \\
\delta \theta & =\frac{r}{2 e}\left(\delta \theta_{l}+\Delta u_{k, l}-\delta \theta_{r}-\Delta u_{k, r}\right),
\end{aligned}
$$

where $r=r_{0}+\Delta r$ is the radius of the rear wheels and $e=e_{0}+\Delta e$ is the distance between them. $\Delta r$ and $\Delta e$ represent the measurement errors for $r_{0}$ and $e_{0}$. The state perturbation $\mathbf{w}_{k}=\left(\Delta \mathbf{u}_{k}^{\mathrm{T}}, \Delta r, \Delta e\right)^{\mathrm{T}}$ accounts thus for all sources of perturbations. The evolution of Minitruck is then described by the state equation equation (1) as

$$
\begin{aligned}
\mathbf{x}_{k+1} & =\mathbf{f}\left(\mathbf{x}_{k}, \mathbf{u}_{k}, \mathbf{w}_{k}\right) \\
= & \left(\begin{array}{c}
x_{k}+\delta s \cdot \cos \left(\theta_{k}+\delta \theta / 2\right) \\
y_{k}+\delta s \cdot \sin \left(\theta_{k}+\delta \theta / 2\right) \\
\theta_{k}+\delta \theta
\end{array}\right)
\end{aligned}
$$

The measurement equation at time $k$ for the $i$-th sonar can then be described as $y_{k, i}=h_{i}\left(\mathbf{x}_{k}\right)+v_{i}$, with $v_{i} \in$ $\left[-\alpha y_{k, i}, \alpha y_{k, i}\right]$. In the obervation equation, $h_{i}\left(\mathbf{x}_{k}\right)$ is the smallest distance between the sensor located at $\mathbf{s}_{i}\left(\mathbf{x}_{k}\right)$ and the nearest segment lying inside the emission cone. All $n_{\mathrm{S}}$ measurement equations may then be gathered to form $\mathbf{h}\left(\mathbf{x}_{k}\right)$. This model allows an easy computation of $\mathbf{h}\left(\mathbf{x}_{k}\right)$, but it is a rather crude description of the range measurement process. Measurements resulting from multiple reflexions of the emitted wave, for instance, may not be explained by this model. They will thus be considered as outliers, which require a specific treatment described in Section IV-A. More details may also be found in [10], [14] and [16].

\section{IMPLEMENTATION ISSUES}

This section describes how outliers, which cannot be avoided when dealing with ultrasonic sensors, can be dealt with in the context of guaranteed state estimation. It also studies the complexity of each part of the localization algorithm, to show its real-time implementability.

\section{A. Robustness to outliers}

For ultrasonic range measurements, sometimes more than $50 \%$ of the data collected turn out to be outliers. These outliers may correspond to an outdated map of the environment, to sensor failures, to people moving in the environment, etc. As a consequence, the set characterized by SIVIA in Section II-D frequently turns out to be empty. To solve this problem, a robust variant of the correction step has to be used.

Each of the $n_{\mathrm{S}}$ sonars of the vehicle provides a range measurement. To the $i$-th measurement $y_{k, i}$, an interval $\left[y_{k, i}\right]=\left[y_{k, i}-\bar{v}_{i}, y_{k, i}-\underline{v}_{i}\right]$ may be associated. This allows the introduction for any given box $[\mathbf{x}]$ of an elementary interval-valued test associated to the $i$-th measurement

$$
\left[t_{i}\right]([\mathbf{x}])= \begin{cases}1 & \text { if }\left[h_{i}\right]([\mathbf{x}]) \subset\left[y_{k, i}\right], \\ 0 & \text { if }\left[h_{i}\right]([\mathbf{x}]) \cap\left[y_{k, i}\right]=\emptyset \\ {[0,1]} & \text { otherwise }\end{cases}
$$

with $\left[h_{i}\right]([\mathbf{x}])$ the $i$-th component of $[\mathbf{h}]([\mathbf{x}])$ and the interval $[0,1]$ corresponding to an inconclusive test. Consider now the sum of all the results of these $n_{\mathrm{S}}$ elementary tests

$$
\begin{aligned}
& \sum_{i=1}^{n_{\mathrm{S}}}\left[t_{i}\right]([\mathbf{x}])=n_{\mathrm{S}} \Leftrightarrow[\mathbf{h}]([\mathbf{x}]) \subset\left[\mathbf{y}_{k}\right], \\
& \sum_{i=1}^{n_{\mathrm{S}}}\left[t_{i}\right]([\mathbf{x}])<n_{\mathrm{S}} \Leftrightarrow[\mathbf{h}]([\mathbf{x}]) \cap\left[\mathbf{y}_{k}\right]=\emptyset .
\end{aligned}
$$

Moreover,

$$
\sum_{i=1}^{n_{\mathrm{S}}}\left[t_{i}\right]([\mathbf{x}]) \geqslant n
$$

implies that any $\mathbf{x} \in[\mathbf{x}]$ is consistent with at least $n$ measurements among the $n_{\mathrm{S}}$ available.

At the beginning of a correction step, the number of outliers is unknown and should be estimated. A first approach would be to characterize the set $\widehat{\mathcal{X}}_{k \mid k}^{\alpha}$ of all $\mathbf{x}_{k}$ that are consistent with $n_{\mathrm{S}}-\alpha$ range measurements using (9), starting with $\alpha=0$ and increasing $\alpha$ by one until $\widehat{\mathcal{X}}_{k \mid k}^{\alpha}$ becomes nonempty. Once such a nonempty set is obtained, one may tolerate one or two more outliers to increase robustness against undetected outliers, at the cost of a deterioration of the precision of the localization. A disadvantage of this technique is that it requires many similar computations to be repeated. A more efficient approach will be presented in Section IV-C.

\section{B. Complexity study}

A worst-case study of the complexity of bounded-error state estimation will now be provided. Recall that the algorithm consists mainly of a prediction and a correction steps.

Correction employs Sivia, the main task of which is the evaluation of $\left[h_{i}\right]([\mathbf{x}])\left(i=1, \ldots, n_{\mathrm{S}}\right)$ for any box $[\mathbf{x}]$ to be tested. This evaluation requires for each sensor $n_{\mathrm{W}}$ distances to be evaluated. To test any box $[\mathbf{x}]$, the complexity is thus linear in the number of sensors and linear in the number 
of segments of the map, which is quite favorable. The bisection process involved by SIVIA makes its complexity exponential with the number of state variables [11], but since the dimension of the state is only three this remains tractable.

When the correction step has to be made robust against outliers, the same localization procedure is simply repeated, each time increasing the number $\alpha$ of tolerated outliers by one. The overall complexity is thus linear with the number of tolerated outliers, which seems again quite acceptable. A way to reduce complexity further is presented in Section IVC.

As regards prediction, the most time-consuming part of IMAGESP is the evaluation of the image of each minced box. Each individual evaluation of the inclusion function is from two to four times more complex than a real-valued function evaluation. Thus, complexity is linear with the number of boxes resulting from the mincing. The number of these boxes critically depends on $\varepsilon_{\mathrm{I}}$, as when $\varepsilon_{\mathrm{I}}$ is halved, the number of minced boxed is multiplied by $2^{\operatorname{dim} \mathbf{x}}$. Merging all image boxes in a single subpaving is also a major time-consuming part of the algorithm. This step, which could be dispensed with, requires a very simple SIVIA algorithm to be employed. The complexity of the merging step is therefore again exponential in the dimension of $\mathrm{x}$, which is still three.

\section{Adaptations for real-time application}

To implement the algorithm in real-time, one has to provide prediction and correction steps that can be executed in a controlled amount of time. Once the number of sensors of the vehicle and segments of the map has been fixed, the complexity for testing any box $[\mathbf{x}]$ in configuration space is fixed. For a given amount of computing time, the number of boxes that may be tested can thus be determined. As a consequence, contrary to applications involving off-line estimation, where the parameter $\varepsilon_{\mathrm{S}}$ is fixed a priori, this parameter has to be evaluated using the volume of the set $\widehat{\mathcal{X}}_{k \mid k-1}$ to be tested at each iteration in order to feed SIVIA with a number boxes to be tested that remains tractable.

Prediction steps are actually performed at a much higher frequency than correction steps because odometric information can be provided much more frequently than telemetric information. To control the computing time required by each prediction step, again the precision parameter $\varepsilon_{\mathrm{I}}$ should be adjusted in such a way that the number of the boxes to be treated remains tractable. Transforming the list of boxes obtained at the second step of IMAGESP into a subpaving should only take place just before the next correction, in order to limit the use of time-consuming SIVIA steps.

To reduce the complexity of the robust estimation technique presented in Section IV-A, one may, instead of repeating the computations for increasing values of $\alpha$, store for each box $[\mathbf{x}]$ tested by SIVIA the number of outliers that should be tolerated to put this box in a robust solution set. Once all boxes have been tested (and their number is bounded by complexity constraints), it is a simple matter to sort out all those that are associated with a given number of tolerated outliers. Time-consuming repeated computations are thus replaced by simple comparisons.

\section{EXAMPLE}

The following example illustrates the ability of this bounded-error localization technique to provide accurate estimates, even in presence of outliers. The model uncertainties are taken as $\Delta r=0.05 r$ and $\Delta e=0.05 e$. The Relative uncertainty parameter $\beta$ is taken as $40 \%$. The policy suggested in Section IV-C to adjust the precision parameters $\varepsilon_{\mathrm{I}}$ and $\varepsilon_{\mathrm{S}}$ was not implemented, and both are taken equal to $5 \mathrm{~cm}$.

Starting from an unknown initial configuration, the bounded-error localization method is able to find where Minitruck is, even when local symmetries complicate the task, see Figure 4. The initial configuration box is $30 \mathrm{~m}$ long, $2.2 \mathrm{~m}$ large, with a $115^{\circ}$ uncertainty on orientation.

The computing time for the initial localization $(k=1)$ is up to 40 seconds in Figure 4, as the initial set to be considered is large. Many configurations were deemed inconsistent with the data. A configuration was deemed inconsistent and eliminated as soon as more data had to be considered as outliers than the minimum required to obtain a non-empty configuration set after correction. Figure 4 shows that at time $k=1$ the set $\widehat{\mathcal{X}}_{1 \mid 1}$ consists of many disconnected components. After the first correction step, computations are performed much faster, as the size of the sets to be considered are smaller. At $k=3$ and $k=4$, measurements allow to eliminate large parts of the set derived from $\widehat{\mathcal{X}}_{1 \mid 1}$ after prediction steps. Only six connected components remained at $k=4$. At time $k=$ 4 , Figure 4 shows that the solution subpaving consists of a single connected component. Between $k=4$ and $k=90$, prediction and correction are performed on this surviving configuration set, which actually contains the correct configuration. Figure 4 displays the projection on the $(X, Y)$-plane of the subpaving obtained at $k=90$, which contains the actual configuration and is contained in the box $[34.35,34.51] \times[1.77,1.81] \times[1.43,1.83]$.

Between $k=50$ and $k=65$, the computing time has been measured. At this stage of the experiment, the uncertainty on the state estimate is small. Results are presented on Figure 5. For this $7.5 \mathrm{~s}$ long experimentation the total computing time, on a $2 \mathrm{GHz}$ Pentium IV processor, is about $5 \mathrm{~s}$ for the bounded-error localization method, which already allows real-time localization.

\section{CONCLUSIONS}

In this paper, a bounded-error state estimation techniques based on interval analysis has been applied to the problem of global vehicle tracking in a mapped environment. Contrary to probabilistic techniques, the proposed algorithm provides at each time instant a set that is guaranteed to contain the actual configuration of the vehicle, provided 

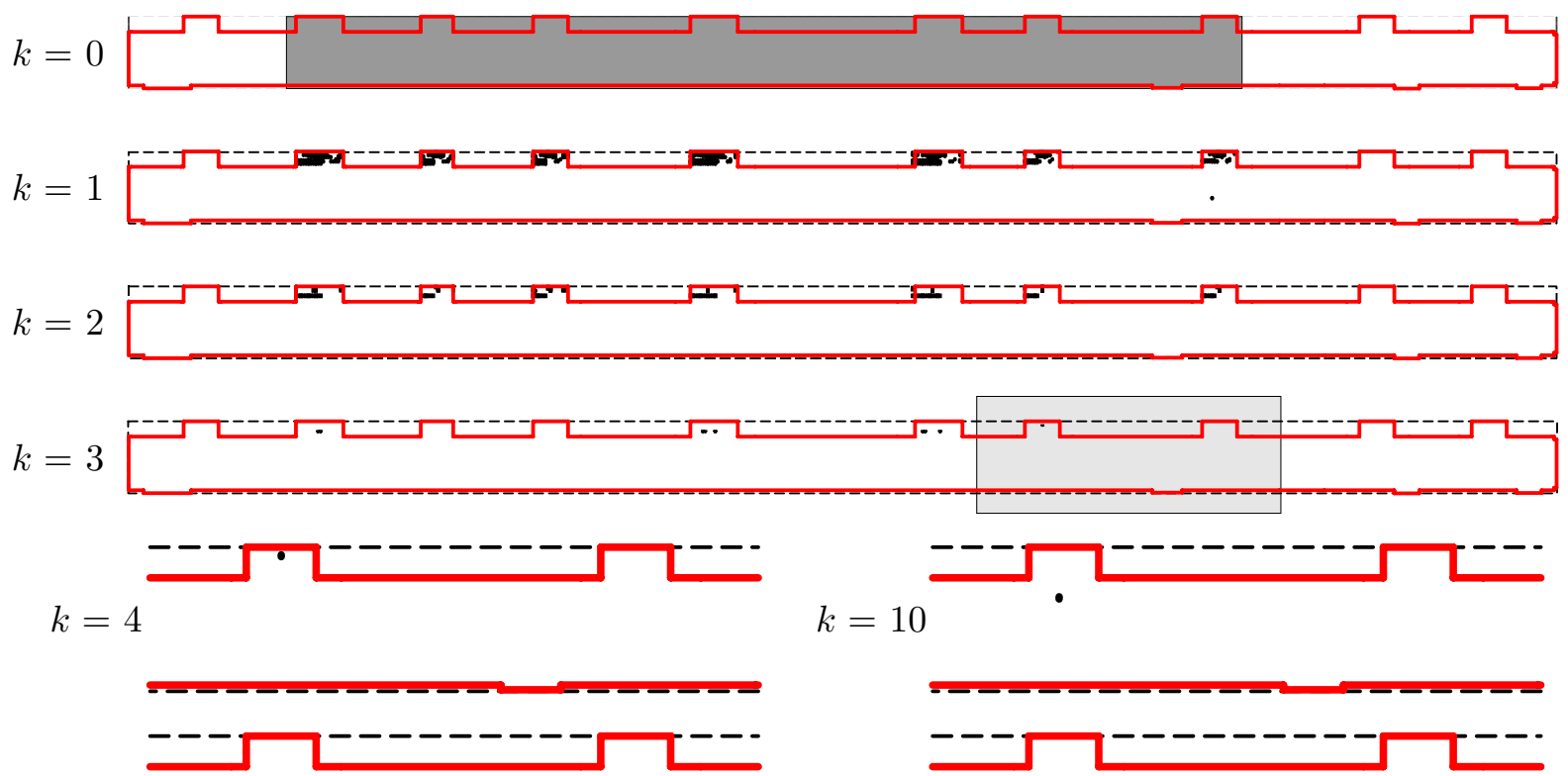

$k=30$

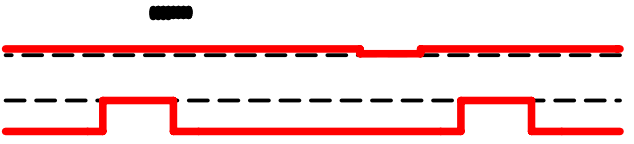

$k=60$

$k=10$

$k=40$

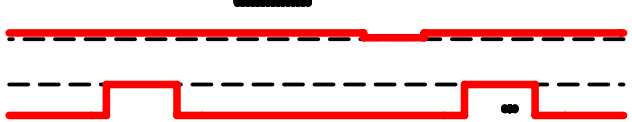

$k=90$

Fig. 4. Bounded-error state estimation with an unknown initial configuration. The last six subfigures correspond to the shaded area represented on the fourth subfigure.

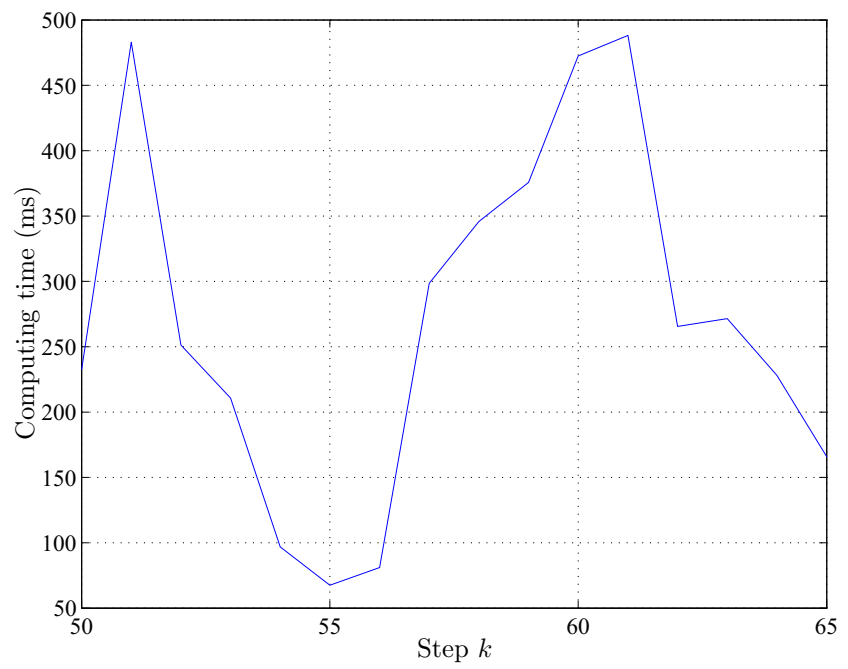

Fig. 5. Computing time

that the assumptions made on the state perturbations and measurement noise and on the number of detected outliers are not violated.

Using odometric and ultrasonic range measurements, it is then possible to achieve multiple hypotheses tracking, as may be required by the ambiguities resulting, e.g., from local symmetries in the environment of the vehicle.

Some material has been provided to facilitate real-time implementation of the algorithm. Experimental results show that the technique is indeed compatible with real-time global tracking.

\section{REFERENCES}

[1] B. Anderson and J. Moore. Optimal Filtering. Prentice-Hall, Englewood Cliffs, 1979.

[2] M. Arulampalam, S. Maskell, N. Gordon, and T. Clapp. A tutorial on particle filters for on-line non-linear/non-gaussian bayesian tracking. IEEE Trans. Signal Processing, 50(2):174-188, 2002.

[3] J. Borenstein, H. Everett, and L. Feng. Navigating Mobile Robots. A. K. Peters Ltd., Wellesley, MA, 1996

[4] W. Burgard, D. Fox, D. Hennig, and T. Schmidt. Estimating the absolute position of a mobile robot using position probability grids. In Proc. Of the Thirteenth National Conference on Artificial Intelligence, pages 896-901, 1996.

[5] I. Cox and G. Wilfong, editors. Autonomous Robot Vehicles. Springer Verlag, 1990.

[6] F. Dellaert, D. Fox, W. Burgard, and S. Thrun. Monte Carlo localisation for mobile robots. In Proc. of the IEEE International Conference on Robotics and Automation, pages 1322-1328, Detroit, MI, 1999.

[7] A. Gelb. Applied Optimal Estimation. MIT Press, Cambridge, MA, 1974.

[8] N. J. Gordon, D. J. Salmond, and A. F. Smith. Novel approach to nonlinear/non-gaussian bayesian state estimation. IEE Procedings $F$, 140(2):107-113, 1993 
[9] L. Jaulin, M. Kieffer, O. Didrit, and E. Walter. Applied Interval Analysis. Springer-Verlag, London, 2001.

[10] L. Jaulin, M. Kieffer, E. Walter, and D. Meizel. Guaranteed robust nonlinear estimation, with application to robot localization. IEEE Transactions on systems, man and cybernetics; Part C-Applications and Reviews, 32(4):374-382, 2003. Accepted.

[11] L. Jaulin and E. Walter. Set inversion via interval analysis for nonlinear bounded-error estimation. Automatica, 29(4):1053-1064, 1993.

[12] M. Kieffer, L. Jaulin, and E. Walter. Guaranteed recursive nonlinear state estimation using interval analysis. In Proceedings of the 37th IEEE Conference on Decision and Control, pages 3966-3971, Tampa, FL, 1998.

[13] M. Kieffer, L. Jaulin, and E. Walter. Guaranteed recursive nonlinear state bounding using interval analysis. International Journal of Adaptative Control and Signal Processing, 6(3):193-218, 2002.

[14] M. Kieffer, L. Jaulin, E. Walter, and D. Meizel. Robust autonomous robot localization using interval analysis. Reliable Computing, 6(3):337-362, 2000.

[15] G. Kitagawa. Monte-Carlo filter and smoother for non-gaussian nonlinear state space models. Journal of Computational and Graphical Statistics, 5(1):1-25, 1996.

[16] D. Meizel, O. Lévêque, L. Jaulin, and E. Walter. Initial localization by set inversion. IEEE Trans. on Robotics and Automation, 18(6):966-971, December 2002.

[17] R. E. Moore. Methods and Applications of Interval Analysis. SIAM, Philadelphia, PA, 1979.

[18] M. Pitt and N. Shephard. Filtering via simulation: Auxiliary particle filters. Journal of the American Statistical Association, 94(446):590599, 1999.

[19] R. Simmons and S. Koenig. Probabilistic robot navigation in partially observable environments. In Proc. International Joint Conference on Artificial Intelligence, 1995.

[20] H. Sorenson. Kalman Filtering: Theory and Application. IEEE Press, 1985.

[21] P. Terwiesch and M. Agarwal. A discretized non-linear state estimator for batch processes. Computers and Chemical Engineering, 19:155$169,1995$.

[22] S. Thrun, D. Fox, W. Burgard, and F. Dellaert. Robust Monte Carlo localization for mobile robots. Artificial Intelligence Journal, 128(12), 2001. 